\title{
Influência da retenção dos anexos fetais no hemograma de fêmeas bovinas da raça Holandesa
}

[The influence of retained fetal membranes on the hemogram of Holstein cows]

J.P.E. Saut, E.H. Birgel Junior*

Faculdade de Medicina Veterinária e Zootecnia - USP

Av. Prof. Dr. Orlando Marques de Paiva, 87

05508-900 - São Paulo, SP

\begin{abstract}
RESUMO
Analisou-se o hemograma de 177 amostras de sangue de fêmeas bovinas da raça Holandesa nos primeiros 90 dias pós-parto. As amostras foram colhidas de vacas não reagentes ao antígeno (GP 51) do vírus da leucose dos bovinos, distribuídas em dois grupos (I: puerpério fisiológico; II: puerpério com retenção dos anexos fetais) e subdivididas em quatro grupos de acordo com o momento de colheita. Nos primeiros 10 dias após o parto, o eritrograma não sofreu influência da retenção dos anexos fetais. Com a evolução do puerpério, observou-se, nas vacas com retenção dos anexos fetais, anemia de grau leve entre o $10^{\circ}$ e $30^{\circ}$ dia pós-parto. O leucograma dos animais com retenção dos anexos fetais foi predominantemente linfocitário, caracterizado por leucopenia, neutropenia com desvio à esquerda degenerativo e eosinopenia. A partir do $10^{\circ}$ dia após o parto, o leucograma não foi influenciado pela retenção dos anexos fetais.
\end{abstract}

Palavras-chave: gado leiteiro, pós-parto, retenção dos anexos fetais, hematologia

\section{ABSTRACT}

Hematological profiles of 177 blood samples from Holstein cows were determined during the first 90 days after parturition. Blood samples were collected from cows that were non-reactive to the bovine leukosis virus (GP 51) antigen. The animals were divided into two groups (group I: physiological puerperium and group II: puerperim with retained fetal membranes), and subdivided into four groups, based on the time that the samples were collected. During the first 10 days after parturition, retained fetal membranes did not influence the erythrogram. However, with the evolution of the puerperium period, a mild anemia was observed from the $10^{\text {th }}$ to the $30^{\text {th }}$ day postpartum in cows with retained fetal membranes. The leukocyte profile was predominantly lymphocytic, characterized by leucopenia induced by neutropenia with a left degenerative shift and eosinopenia. From the $10^{\text {th }}$ day postpartum, the retained fetal membranes did not influence the leukogram

Keywords: dairy cow, postpartum, retained fetal membranes, hematology

\section{INTRODUÇÃO}

Dentre os distúrbios que acometem os animais no puerpério, um dos problemas mais freqüentemente diagnosticados em vacas leiteiras é a retenção dos anexos fetais (Wischral et al., 2001), sendo essa enfermidade responsável por perda econômica (Mulei et al., 1993) decorrente de distúrbios de fertilidade. A incidência de retenção dos anexos em um rebanho bovino livre de brucelose pode variar, em vacas leiteiras, entre 3 e 12\% (Smith, 1993). Estudo realizado na Região Sul de Minas Gerais mostrou incidência de $10,5 \%$ de retenção dos anexos fetais em 1.875 partos avaliados (Fernandes et al., 2001).

Afora prejuízos relativos à performance reprodutiva, como maior incidência de infecção

Recebido em 4 de setembro de 2006

Aceito em 21 de julho de 2008

* Autor para correspondência (corresponding author)

E-mail: ehbirgel@usp.br 
uterina 30 dias pós-parto, maior intervalo entre parto e primeiro cio, maior número de serviço/concepção e maior intervalo entre partos (Fernandes et al., 2001), a retenção dos anexos fetais resultou em modificações na crase sangüínea dos bovinos, sendo relatado em publicações referentes à influência dessa condição patológica no hemograma (Moore, 1946; Straub et al., 1959; Mulei et al., 1993; Cai et al., 1994; Gonçalves e Kozicki, 1997; Srinivas et al., 1999), principalmente, a ocorrência de leucopenia e neutropenia nos primeiros dias de puerpério.

Nessas pesquisas verificou-se que o quadro hemático não foi acompanhado, detalhadamente, durante toda a evolução do puerpério, encontrando-se duas pesquisas (Gonçalves e Kozicki, 1997; Srinivas, 1999) em que as colheitas se limitaram ao primeiro dia do puerpério, três que realizaram esse acompanhamento nos sete primeiros dias do puerpério (Moore, 1946; Straub et al., 1959;
Mulei et al., 1993) e uma que o realizou semanalmente (Cai et al., 1994). Deve ser salientado, ainda, que apenas a pesquisa de Gonçalves e Kozicki (1997) foi realizada em condições brasileiras de clima e manejo.

Assim, a presente pesquisa teve a finalidade de avaliar a influência da retenção dos anexos fetais sobre os valores do quadro hemático de bovinos da raça Holandesa.

\section{MATERIAL E MÉTODOS}

No período entre a parição e 90 dias pós-parto, foram colhidas 177 amostras de sangue de fêmeas bovinas da raça Holandesa, provenientes de três propriedades produtoras do leite tipo A e oito do tipo B, livres de brucelose e tuberculose, e não reagentes ao antígeno glicoprotéico (gp 51) do vírus da leucose dos bovinos. As amostras de sangue utilizadas para a avaliação da influência da retenção dos anexos fetais foram agrupadas conforme a Tab. 1 .

Tabela 1. Constituição dos grupos experimentais para avaliar a influência da retenção dos anexos fetais no hemograma de vacas da raça Holandesa

\begin{tabular}{cccc}
\hline Grupo & Tempo de colheita de sangue & Animais sadios & $\begin{array}{c}\text { Animais com retenção } \\
\text { dos anexos fetais }\end{array}$ \\
\hline I & $\begin{array}{c}1^{\circ}-\mid 4^{\circ} \text { dia pós-parto } \\
(24-\mid 96 \text { horas pós-parto })\end{array}$ & 17 & 15 \\
II & $4^{\circ}-\mid 10^{\circ}$ dia pós-parto & 36 & 14 \\
III & $(96-\mid 240$ horas pós-parto $)$ & 16 & 12 \\
& $(240-\mid 720$ horas pós-parto $)$ & & 18 \\
IV & $30^{\circ}-\mid 90^{\circ}$ dia pós-parto & 49 & \\
& $(720-\mid 2160$ horas pós-parto $)$ & 49 & \\
\hline
\end{tabular}

O sangue foi colhido por punção da veia jugular externa, em tubos siliconizados, contendo uma solução aquosa de EDTA-K $\mathrm{K}_{3}$ a $15 \%$. Nas amostras de sangue foram realizadas as seguintes provas: contagem do número de hemácias, em câmara de Neubauer modificada, utilizando líquido de Gower como diluidor; determinação do volume globular, pelo método do microematócrito; dosagem de hemoglobina, pelo método de cianometaemoglobina, cálculo dos índices hematimétricos absolutos (VCM, HCM, CHCM); contagem do número total de leucócitos, em câmara de Neubauer modificada, utilizando-se o líquido de Thoma como diluidor e a contagem diferencial de leucócitos, efetuada em esfregaços sangüíneos confeccionados com sangue in natura e corados pelo método pancromático de Rosenfeld. As técnicas empregadas na determinação do hemograma seguiram as recomendações de Birgel (1982).

Em cada esfregaço sangüíneo, foram diferenciados 100 leucócitos e classificados de acordo com suas características morfológicas e tintoriais, em neutrófilos com núcleo em bastonete, neutrófilos com núcleo segmentado, eosinófilos, basófilos, linfócitos e monócitos.

Para avaliar a influência da retenção dos anexos fetais no hemograma, utilizou-se o programa de 
computador SAS, aplicando-se, inicialmente, a análise de variância e, a seguir, a comparação entre os pares de médias pelo teste Tukey, com nível de significância igual a 5\% (Berquó et al., 1981).

\section{RESULTADOS}

Nos primeiros 10 dias após o parto, o número de hemácias não sofreu influência da retenção dos anexos fetais, porém, com a evolução do puerpério (entre $10-30$ dias após o parto) observou-se nas vacas com retenção dos anexos fetais que o número de hemácias foi menor que o observado em animais sadios (Tab. 2).

A taxa de hemoglobina, o volume globular, o volume corpuscular médio (VCM), a hemoglobina corpuscular média (HCM) e a concentração de hemoglobina corpuscular média (CHCM) não foram influenciadas pela retenção dos anexos fetais (Tab. 2).

O quadro leucocitário de vacas com retenção dos anexos fetais foi caracterizado por leucopenia por neutropenia com desvio à esquerda degenerativo, eosinopenia. A partir do $10^{\circ}$ dia pós-parto, o leucograma deixa de sofrer influência da retenção dos anexos fetais (Tab. 3, 4 e Fig. 1). Nas amostras de sangue colhidas, nos quatro primeiros dias do puerpério, observou-se que nos animais com retenção dos anexos fetais o número de leucócitos circulantes era cerca de 4.000 células, mais baixo que o observado nos animais sadios, sendo os polimorfonucleares neutrófilos os principais responsáveis pelas modificações observadas nesse período.

Tabela 2. Média e desvio-padrão dos constituintes do eritrograma de bovinos da raça Holandesa, nos primeiros 90 dias pós-parto, distribuídos segundo a influência da retenção dos anexos fetais

\begin{tabular}{|c|c|c|c|c|c|}
\hline \multirow{2}{*}{\multicolumn{2}{|c|}{ Eritrograma }} & \multicolumn{4}{|c|}{ Dias pós-parto } \\
\hline & & \multirow{2}{*}{$\begin{array}{l}1^{\circ}-\mid 4^{\circ} \text { dia } \\
5,57 \pm 0,73 a\end{array}$} & \multirow{2}{*}{$\begin{array}{c}4^{\circ}-10^{\circ} \text { dia } \\
5,64 \pm 0,86 a\end{array}$} & \multirow{2}{*}{$\frac{10^{\circ}-\mid 30^{\circ} \text { dia }}{5,66 \pm 1,14 \mathrm{a}}$} & \multirow{2}{*}{$\begin{array}{c}30^{\circ}-\mid 90^{\circ} \text { dia } \\
5,39 \pm 0,68 \mathrm{a}\end{array}$} \\
\hline Hemácias & Fisiológico & & & & \\
\hline$\left(\mathrm{x} 10^{6} / \mu \mathrm{l}\right)$ & Retenção & $5,59 \pm 0,69 \mathrm{a}$ & $5,37 \pm 0,69 a$ & $4,78 \pm 0,94 b$ & $5,20 \pm 0,71 \mathrm{a}$ \\
\hline \multirow{2}{*}{$\begin{array}{l}\text { Hemoglobina } \\
\text { (g/dl ) }\end{array}$} & Fisiológico & $10,60 \pm 1,09 \mathrm{a}$ & $10,41 \pm 1,07 \mathrm{a}$ & $10,22 \pm 1,33 a$ & $10,04 \pm 1,30 \mathrm{a}$ \\
\hline & Retenção & $10,71 \pm 0,90 \mathrm{a}$ & $9,93 \pm 0,86 a$ & $9,31 \pm 1,26 \mathrm{a}$ & $9,84 \pm 1,05 \mathrm{a}$ \\
\hline \multirow{2}{*}{$\begin{array}{l}\text { Volume globular } \\
(\%)\end{array}$} & Fisiológico & $29,3 \pm 3,2 \mathrm{a}$ & $29,0 \pm 2,9 \mathrm{a}$ & $28,4 \pm 3,9 \mathrm{a}$ & $28,2 \pm 3,6 a$ \\
\hline & Retenção & $30,3 \pm 2,8 \mathrm{a}$ & $27,5 \pm 2,3 \mathrm{a}$ & $26,7 \pm 4,0 \mathrm{a}$ & $27,6 \pm 2,6 a$ \\
\hline \multirow{2}{*}{$\begin{array}{l}\mathrm{VCM} \\
(\mathrm{fl})\end{array}$} & Fisiológico & $53,09 \pm 7,24 a$ & $52,14 \pm 6,99 a$ & $51,39 \pm 8,88 \mathrm{a}$ & $53,17 \pm 7,67 a$ \\
\hline & Retenção & $51,17 \pm 6,83 a$ & $51,72 \pm 5,62 \mathrm{a}$ & $57,16 \pm 9,92 \mathrm{a}$ & $53,62 \pm 6,04 \mathrm{a}$ \\
\hline \multirow{2}{*}{$\begin{array}{c}\mathrm{HCM} \\
(\mathrm{pg})\end{array}$} & Fisiológico & $19,25 \pm 2,88 \mathrm{a}$ & $18,66 \pm 2,12 \mathrm{a}$ & $18,41 \pm 2,69 \mathrm{a}$ & $18,94 \pm 3,01 \mathrm{a}$ \\
\hline & Retenção & $18,04 \pm 1,98 \mathrm{a}$ & $18,70 \pm 2,27 \mathrm{a}$ & $19,96 \pm 3.83 \mathrm{a}$ & $19,08 \pm 2,22 \mathrm{a}$ \\
\hline \multirow{2}{*}{$\begin{array}{c}\mathrm{CHCM} \\
(\%)\end{array}$} & Fisiológico & $36,28 \pm 2,02 \mathrm{a}$ & $35,98 \pm 2,51 \mathrm{a}$ & $36,01 \pm 1,58 \mathrm{a}$ & $35,62 \pm 2,46 a$ \\
\hline & Retenção & $35,39 \pm 1,87 \mathrm{a}$ & $36,17 \pm 1,42 \mathrm{a}$ & $34,85 \pm 1,44 \mathrm{a}$ & $35,62 \pm 1,57 \mathrm{a}$ \\
\hline
\end{tabular}

Valores seguidos por letras distintas indicam diferença estatística entre vacas com puerpério físiológico e retenção dos anexos fetais pelo teste Tukey $(\mathrm{P} \leq 0,05)$. VCM: volume corpuscular médio; HCM: hemoglobina corpuscular média; CHCM: concentração de hemoglobina corpuscular média.

Nas amostras de sangue colhidas entre $1-\mid 4$ dias e 4 - 10 dias após o parto, constatou-se que o número total de neutrófilos obtidos nos animais com retenção dos anexos fetais foi estatisticamente mais baixo que os valores encontrados nos animais com puerpério fisiológico. $\mathrm{Na}$ fase inicial do puerpério, especificamente nas amostras colhidas entre os dias $4-\mid 10$, houve diferença no número absoluto de neutrófilos com núcleo em forma de bastonete. Em relação ao número absoluto de neutrófilos com núcleo segmentado, as variações observadas foram semelhantes às descritas para 0 número total de neutrófilos. Nos animais com retenção dos anexos fetais, observou-se que os neutrófilos com núcleo segmentado representaram entre $85,8 \%$ e $99,7 \%$ dos tipos de neutrófilos encontrados nos diversos grupos 
experimentais, enquanto os neutrófilos com núcleo em forma de bastonete representaram entre $0,3 \%$ e $14,2 \%$ de todos os neutrófilos observados no período estudado. Não foram encontradas na circulação, em nenhuma ocasião, células imaturas dessa série de granulócitos.

Quanto ao número de linfócitos, o quadro leucocitário nos animais com retenção dos anexos fetais, à semelhança do verificado nos animais com puerpério fisiológico, foi predominantemente linfocitário. $\mathrm{O}$ número absoluto de linfócitos não sofreu influência da retenção dos anexos fetais (Tab. 3).

Nas amostras colhidas entre $1-4$ dias após o parto, foi observado que o número absoluto de eosinófilos obtidos nos animais com retenção dos anexos fetais foi estatisticamente mais baixo que os valores obtidos nos animais com puerpério fisiológico.

O número absoluto de basófilos não sofreu influência da retenção dos anexos fetais. Em relação ao número absoluto de monócitos, nas amostras colhidas entre $1-4$ dias após o parto o valor foi mais baixo nos animais com retenção dos anexos fetais que o obtido nos animais com puerpério fisiológico. Também, observou-se diferença significativa nas amostras colhidas entre $30-90$ dias após o parto, sendo o número absoluto de monócitos nos animais com retenção dos anexos fetais mais elevado do que o valor observado nos animais com puerpério fisiológico.

Tabela 3. Média e desvio-padrão dos constituintes do leucograma de bovinos da raça Holandesa, nos primeiros 90 dias pós-parto, distribuídos segundo a influência da retenção dos anexos fetais

\begin{tabular}{|c|c|c|c|c|c|}
\hline \multirow{2}{*}{\multicolumn{2}{|c|}{ Leucograma }} & \multicolumn{4}{|c|}{ Dias pós-parto } \\
\hline & & \multirow{2}{*}{$\frac{1^{\circ}-\mid 4^{\circ} \mathrm{dia}}{14.432 \pm 4.710 \mathrm{a}}$} & \multirow{2}{*}{$\frac{4^{\circ}-10^{\circ} \mathrm{dia}}{13.318 \pm 5.257 \mathrm{a}}$} & \multirow{2}{*}{$\frac{10^{\circ}-30^{\circ} \mathrm{dia}}{11.113 \pm 3.571 \mathrm{a}}$} & \multirow{2}{*}{$\frac{30^{\circ}-190^{\circ} \mathrm{dia}}{10.849 \pm 2.526 a}$} \\
\hline Leucócitos & Fisiológico & & & & \\
\hline$\left(\right.$ células $/ \mathrm{mm}^{3}$ ) & Retenção & $10.417 \pm 1.416 \mathrm{~b}$ & $12.682 \pm 5.694 \mathrm{a}$ & $11.079 \pm 4.455 \mathrm{a}$ & $11.419 \pm 3.655 \mathrm{a}$ \\
\hline \multirow{2}{*}{$\begin{array}{l}\text { Neutrófilos } \\
\text { (células/mmª) }\end{array}$} & Fisiológico & $5.024 \pm 2.521 \mathrm{a}$ & $4.573 \pm 3.371 \mathrm{a}$ & $3.352 \pm 1.676 \mathrm{a}$ & $3.410 \pm 1.333 \mathrm{a}$ \\
\hline & Retenção & $2.558 \pm 1.837 b$ & $2.511 \pm 1.347 b$ & $3.038 \pm 1.823 \mathrm{a}$ & $3.363 \pm 1.506 \mathrm{a}$ \\
\hline \multirow{2}{*}{$\begin{array}{l}\text { Eosinófilos } \\
\text { (células/mm³) }\end{array}$} & Fisiológico & $311 \pm 304 a$ & $218 \pm 312 \mathrm{a}$ & $515 \pm 661 \mathrm{a}$ & $575 \pm 539 a$ \\
\hline & Retenção & $123 \pm 153 b$ & $133 \pm 178 \mathrm{a}$ & $579 \pm 582 a$ & $453 \pm 389 a$ \\
\hline \multirow{2}{*}{$\begin{array}{c}\text { Basófilos } \\
\text { (células/mm³) }\end{array}$} & Fisiológico & $54 \pm 128 \mathrm{a}$ & $56 \pm 122 \mathrm{a}$ & $24 \pm 46 a$ & $78 \pm 104 a$ \\
\hline & Retenção & $22 \pm 47 \mathrm{a}$ & $27 \pm 70 \mathrm{a}$ & $18 \pm 48 \mathrm{a}$ & $43 \pm 68 a$ \\
\hline \multirow{2}{*}{$\begin{array}{c}\text { Linfócitos } \\
\text { (células/ mm³) }\end{array}$} & Fisiológico & $8.791 \pm 3.605 a$ & $8.394 \pm 3.512 \mathrm{a}$ & $7.215 \pm 2.774 \mathrm{a}$ & $6.725 \pm 2.114 a$ \\
\hline & Retenção & $7.679 \pm 1.522 \mathrm{a}$ & $9.909 \pm 5.068 \mathrm{a}$ & $7.309 \pm 3.979 \mathrm{a}$ & $7.408-3.881 \mathrm{a}$ \\
\hline \multirow{2}{*}{$\begin{array}{c}\text { Monócitos } \\
(\text { células/mm²) }\end{array}$} & Fisiológico & $164 \pm 194 a$ & $74 \pm 164 a$ & $45 \pm 76 a$ & $58 \pm 100 \mathrm{a}$ \\
\hline & Retenção & $34 \pm 94 b$ & $103 \pm 94 \mathrm{a}$ & $166 \pm 228 \mathrm{a}$ & $142 \pm 152 b$ \\
\hline
\end{tabular}

$\overline{\text { Valores seguidos por letras distintas indicam diferença estatística entre vacas com puerpério fisiológico e retenção }}$ dos anexos fetais pelo teste Tukey $(\mathrm{P} \leq 0,05)$.

Tabela 4. Média e desvio-padrão do número absoluto de neutrófilos de bovinos da raça Holandesa, nos primeiros 90 dias pós-parto, distribuídos segundo a influência da retenção dos anexos fetais

\begin{tabular}{cccccc}
\multicolumn{2}{c}{ Neutrófilos } & $1^{\mathrm{o}}-14^{\circ} \mathrm{dia}$ & $4^{\circ}-110^{\circ}$ dia & $10^{\circ}-130^{\circ}$ dia & $30^{\circ}-190^{\circ}$ dia \\
\hline $\begin{array}{c}\text { Bastonete } \\
\left(\text { células } / \mathrm{mm}^{3}\right)\end{array}$ & Fisiológico & $152 \pm 169 \mathrm{a}$ & $102 \pm 175 \mathrm{a}$ & $16 \pm 45 \mathrm{a}$ & $1 \pm 10 \mathrm{a}$ \\
& Retenção & $252 \pm 252 \mathrm{a}$ & $356 \pm 454 \mathrm{~b}$ & $30 \pm 85 \mathrm{a}$ & $9 \pm 39 \mathrm{a}$ \\
Segmentado & Fisiológico & $4.872 \pm 2.533 \mathrm{a}$ & $4.471 \pm 3.303 \mathrm{a}$ & $3.336 \pm 1.655 \mathrm{a}$ & $3.408 \pm 1.334 \mathrm{a}$ \\
$\left(\right.$ células $\left./ \mathrm{mm}^{3}\right)$ & Retenção & $2.307 \pm 1.831 \mathrm{~b}$ & $2.154 \pm 1.123 \mathrm{~b}$ & $3.008 \pm 1.841 \mathrm{a}$ & $3.354 \pm 1.493 \mathrm{a}$ \\
& & & & & \\
Total & Fisiológico & $5.024 \pm 2.521 \mathrm{a}$ & $4.573 \pm 3.371 \mathrm{a}$ & $3.352 \pm 1.676 \mathrm{a}$ & $3.410 \pm 1.333 \mathrm{a}$ \\
$\left(\right.$ células $\left./ \mathrm{mm}^{3}\right)$ & Retenção & $2.558 \pm 1.837 \mathrm{~b}$ & $2.511 \pm 1.347 \mathrm{~b}$ & $3.038 \pm 1.823 \mathrm{a}$ & $3.363 \pm 1.506 \mathrm{a}$ \\
\hline
\end{tabular}

Valores seguidos por letras distintas indicam diferença estatística entre vacas com puerpério fisiológico e retenção dos anexos fetais pelo teste Tukey $(\mathrm{P} \leq 0,05)$. 
Influência da retenção...

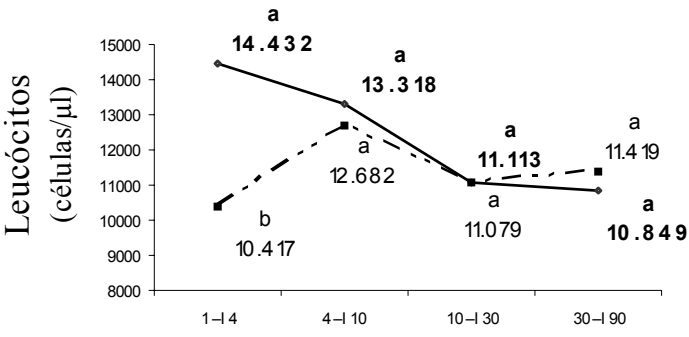

Dias pós-parto

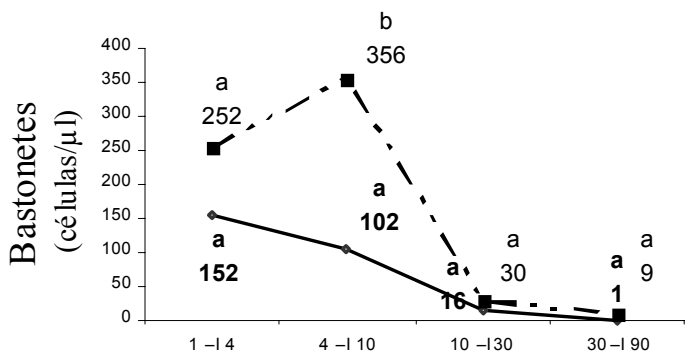

Dias pós-parto

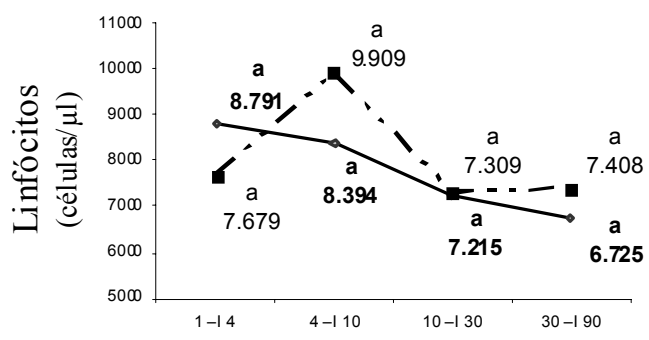

Dias pós-parto

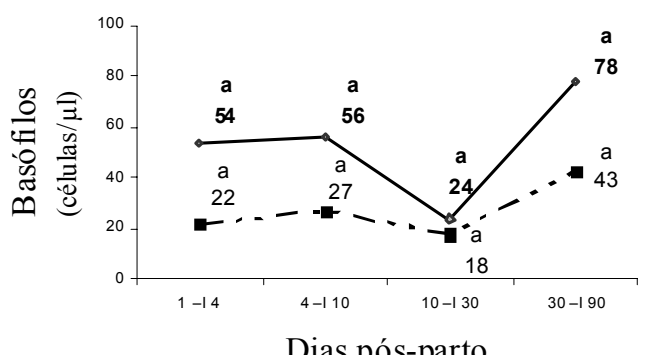

Dias pós-parto

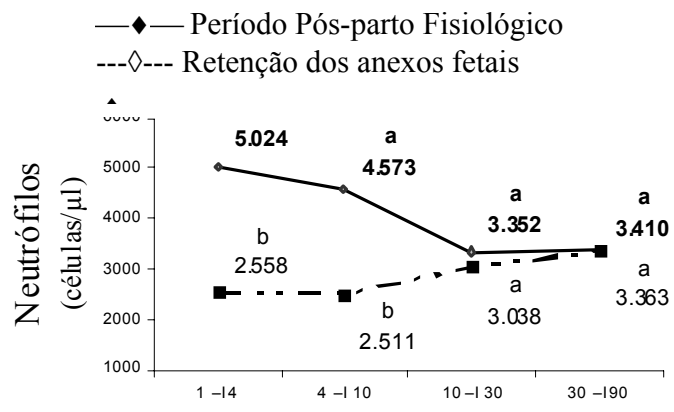

Dias pós-parto

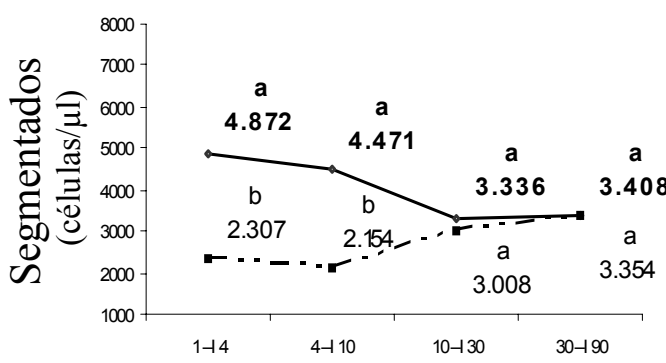

Dias pós-parto

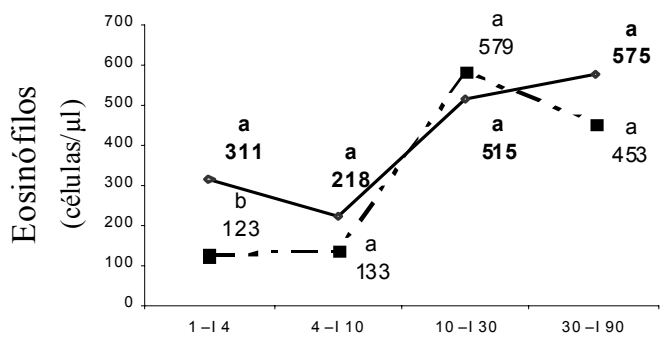

Dias pós-parto

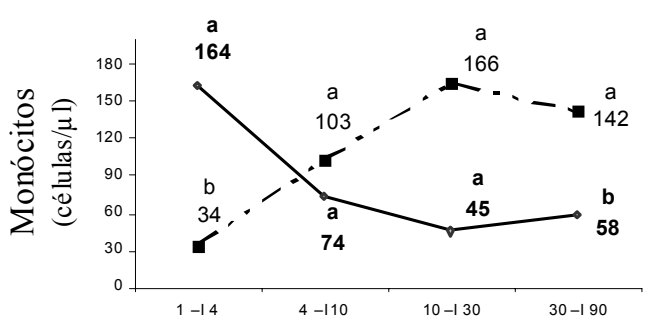

Dias pós-parto

Valores seguidos por letras distintas indicam diferença estatística entre vacas com puerpério fisiológico e retenção dos anexos fetais pelo teste de Tukey $(\mathrm{P} \leq 0,05)$

Figura 1. Valores médios do leucograma de bovinos da raça Holandesa, nos primeiros 90 dias pós-parto, distribuídos segundo a influência da retenção dos anexos fetais. 


\section{DISCUSSÃO E CONCLUSÃO}

O presente trabalho demonstrou que, em relação aos constituintes do eritrograma, exceção feita ao número de hemácias, não houve diferença significativa entre animais com retenção dos anexos fetais de animais sadios, nos primeiros 10 dias de puerpério. Estas observações são semelhantes às dos autores que estudaram a influência da retenção dos anexos fetais sobre o número de hemácias (Moore, 1946; Straub et al., 1959; Mulei et al., 1993), a taxa de hemoglobina (Straub et al., 1959; Mulei et al., 1993) e o volume globular (Straub et al., 1959; Mulei et al., 1993), e não observaram variações no eritograma associadas a essa condição, exceção feita à pesquisa de Srinivas et al. (1999). Em relação ao VCM, HCM e CHCM, não foram encontrados trabalhos que pudessem ser utilizados para comparação dos resultados. Aparentemente, esta foi a primeira vez que se pesquisou e demonstrou que os índices hematimétricos não foram influenciados pela retenção dos anexos fetais durante o puerpério recente. Diante da ausência de outras referências a uma possível influência da retenção dos anexos fetais sobre o número de hemácias, recomenda-se que outras pesquisas sejam realizadas para esclarecer, em definitivo, a natureza dessa influência, isto é, se com a evolução do quadro clínico ocorre anemia de grau leve entre o $10^{\circ}$ e $30^{\circ}$ dia de puerpério.

Sob o ponto de vista hematológico, a retenção dos anexos fetais foi responsável por alterações no leucograma, demonstrando que a medula não conseguia responder de forma satisfatória aos estímulos do processo de doença e /ou toxiinfeccioso instalado.

$\mathrm{O}$ número total de neutrófilos e o número absoluto de neutrófilos segmentado foram os principais responsáveis pelas modificações observadas nesse período, no leucograma de animais com retenção dos anexos fetais. Os resultados desta pesquisa são semelhantes aos apresentados por Moore (1946), Straub et al. (1959) e Cai et al. (1994), que descreveram a existência de neutropenia relativa nos animais com retenção dos anexos fetais, e diferem dos relatados por Mulei et al. (1993), que não encontraram diferenças nos valores relativos de neutrófilos nessa fase inicial do puerpério. Segundo Gonçalves e Kozicki (1997), na única pesquisa na qual o número absoluto de neutrófilos foi determinado, nas amostras de sangue colhidas imediatamente após o parto, já era possível observar que o número total de leucócitos e o número absoluto de neutrófilos nos animais que retiveram os anexos fetais era menor que o observado nos animais sadios.

Ao caracterizar o quadro leucocitário nos animais com retenção dos anexos fetais, Moore (1946) e Straub et al. (1959) relataram a existência de aumento de formas jovens de neutrófilos (núcleo em forma de bastonete, metamielócito e mielócito), caracterizado como um desvio à esquerda, além de um tipo de resposta mielóide escalonada, fato relatado anteriormente por Straub et al. (1959) e Mulei et al. (1993). Pode-se verificar este evento no grupo composto por animais entre $4-10$ dias de evolução do puerpério, porém sem a presença de formas imaturas de neutrófilos, como os metamielócitos e mielócitos. Estes resultados permitiram caracterizar, nos primeiros dias após o parto, a ocorrência de desvio à esquerda degenerativo, no qual se observou neutropenia com aumento do número de formas jovens de neutrófilos

O quadro leucocitário observado nesse período, nos animais com retenção dos anexos fetais, foi semelhante àquele observado em casos de esgotamento do tecido mielóide, ou seja, a retenção dos anexos fetais associado à endometrite puerperal aguda leva a uma depleção da medula óssea. Segundo Moore (1946), essas alterações eram semelhantes às observadas em condições de severa toxemia ou após a administração de venenos e drogas que tenham ação seletiva sobre a medula óssea. No caso da retenção dos anexos fetais, segundo o autor, a depressão da medula seria decorrente da absorção de toxinas oriundas da decomposição desses.

Os resultados obtidos nesta pesquisa permitiram supor que a depleção da medula óssea estaria relacionada à mencionada inibição da medula, conseqüente do processo toxiinfeccioso instalado, impedindo a adequada resposta leucocitária ou, então, que os neutrófilos produzidos ou reunidos nos compartimentos de reserva, diante da endometrite associada à retenção dos anexos fetais, determinariam a migração dessas células para os tecidos uterinos inflamados. 
A descrição das alterações observadas no período inicial do puerpério mostrou que, nas vacas com retenção dos anexos fetais o número absoluto de eosinófilos foi estatisticamente menor do que o obtido nos animais com puerpério fisiológico. Estes resultados assemelham-se aos de Straub et al. (1959) e Mulei et al. (1993) que relataram haver diferença significativa nos cinco dias iniciais do puerpério, e diferem dos de Moore (1946), que não verificou variações no número de eosinófilos nos animais com retenção dos anexos fetais, da parição até 192 horas pós-parto. Ressalta-se que a ocorrência de eosinopenia durante a fase neutrofílica foi uma das características observadas na fase inicial do processo toxiinfeccioso, sendo esse fenômeno evidenciado nos resultados obtidos na presente pesquisa e referido na literatura sobre hematologia (Birgel, 1982).

O leucograma não foi influenciado pela retenção dos anexos fetais a partir do $10^{\circ}$ dia do puerpério, e na única pesquisa que avaliou esse fato, Moore (1946) observou que o quadro leucocitário havia normalizado 196 horas pós-parto.

No período avaliado observou-se que o quadro leucocitário nas fêmeas bovinas com retenção dos anexos fetais foi, predominantemente, linfocitário, e em nenhum momento estudado observou-se influência dessa retenção. Straub et al. (1959) observaram que o número de linfócitos nos animais com retenção dos anexos fetais era mais baixo e Cai et al. (1994) verificaram que as vacas com retenção dos anexos fetais apresentavam maior número de linfócitos circulantes.

Valores de referência obtidos de bovinos no estado de São Paulo (Birgel Junior, 1991; Costa, 1994; Távora, 1997) não permitiram, apesar das diferenças estatísticas encontradas, demonstrar a existência de um quadro de monocitose nos animais com retenção dos anexos fetais, sendo que a possível influência da retenção sobre o número absoluto de monócitos não está caracterizada na literatura. Moore (1946) não observou desvio nos valores relativos de monócitos, do momento da parição até 192 horas após o parto, enquanto Straub et al. (1959) concluíram que ocorreu monocitose absoluta associada tanto a animais com puerpério fisiológico quanto a animais com retenção.

\section{REFERÊNCIAS BIBLIOGRÁFICAS}

BERQUÓ, E.S.; SOUZA, J.M.P.; GOTLIEB, S.L.D. (Eds). Bioestatística. São Paulo: Pedagógica e Universitária, 1980. 325p.

BIRGEL, E.H. Hematologia clínica veterinária. In: BIRGEL, E.H.; BENESI, F.J. (Eds). Patologia clínica veterinária. 2.ed. São Paulo: SPMV, 1982. 260p.

BIRGEL JUNIOR, E.H. Hemograma de bovinos (Bos taurus, Lennaeus, 1758) da raça Jersey, criados no estado de São Paulo. Influência de fatores etários, sexuais e da infecção por vírus da leucose bovina. 1991. 172f. Dissertação (Mestrado) - Faculdade de Medicina Veterinária e Zootecnia, Universidade de São Paulo, São Paulo.

CAI, T.Q.; WESTON, P.G.; LUND, L.A. et al. Association between neutrophil functions and periparturient disorders in cows. Am. J. Vet. Res., v.55, p.934-943, 1994.

COSTA, J.N. Leucograma de Zebuinos (Bos indicus, Linnaeus, 1758) sadios da raça Nelore, criados no estado de São Paulo. Influência de fatores etários e sexuais. 1994. 124f. Dissertação (Mestrado) - Faculdade de Medicina Veterinária e Zootecnia, Universidade de São Paulo, São Paulo.

FERNANDES, C.A.C.; COSTA, D.S.; VIANA, J.H.M. Impacto da retenção de placenta sobre a performance reprodutiva de vacas leiteiras. Rev. Bras. Reprod. Anim., v.25, p.26-30, 2001.

GONÇALVES, D.; KOZICKI, L.E. Perfis bioquímicos e imunológicos no período peripartal de vacas leiteiras com e sem retenção de placenta. Braz. Journal Vet. Res. Anim. Sci., v.34, p.364-370, 1997.

MOORE, B.A. The blood picture in cases of retained fetal membranes in cattle. J. Am. Vet. Med. Assoc., v.109, p.39-45, 1946.

MULEI, C.M.; MUTIGA, E.R.; GITAU, G.K. Retained foetal membranes in cattle: relationship between bacterial isolates, blood picture and systemic involvement. Indian J. Anim. Sci., v.63, p.235-238, 1993.

SMITH, B.P. (Ed). Tratado de medicina interna de grandes animais. São Paulo: Manole, 1993. p.258-260. 
SRINIVAS, T.; NAIDU, K.S.; BRAHMAIAH, K.V. et al. Retained foetal membranes in crossbred cows a haematological and biochemical study. Indian Vet. J., v.76, p.233$235,1999$.

STRAUB, O.C.; SCHALM, O.W.; HUGHES, J.P. et al. Bovine hematology. II. Effect of parturition and retention of fetal membranes on blood morphology. J. Am. Vet. Med. Assoc., v.15, p.618-622, 1959.

TÁVORA, J.P.F. Hemograma de bovinos das raças Gir, Girolando e Holandesa criados no estado de São Paulo. Estabelecimento dos valores de referência e avaliação da influência de fatores de variabilidade raciais, etários e sexuais. 1998. 163f. Tese (Doutorado) - Faculdade de Medicina Veterinária e Zootecnia, Universidade de São Paulo, São Paulo.

USER'S guide: statistics. Cary, NC: SAS Institute, 1985. 956p.

WISCHRAL, A.; NISHIYAMA-NARUKE, A.; CURI, B. et al. Plasma concentrations of estradiol $17 \beta$ and PGF2 $\alpha$ metabolite and placental fatty acid composition and antioxidant enzyme activity in cows with and whithout retained fetal membranes. Prostagl. Lipid Mediat., v.65, p.117-124, 2001. 